\title{
Characterization of Break in Quantum Grid
}

\author{
M. ArChiBAlD ${ }^{a, *}$, S. CURriE ${ }^{a}$ AND M. NOWACZYK ${ }^{b}$ \\ ${ }^{a}$ The John Knopfmacher Center for Applicable Analysis and Number Theory, \\ University of the Witwatersrand, Johannesburg, South Africa \\ ${ }^{b}$ AGH University of Science and Technology, Kraków, Poland
}

Received: 31.10.2019 \& Accepted: 10.06.2020

Doi: $10.12693 /$ APhysPolA.138.383

*e-mail: margaret.archibald@wits.ac.za

\begin{abstract}
A finite rectangular grid can be used to model many applications in physics and related areas, where finite periodic orbits on this grid can be altered if a break occurs on the grid. For a break at a node, it is useful to be able to identify the position and type of the break without having to examine the whole grid. By calculating how much the sum of the amplitude numbers over all periodic orbits changes in the event of a break for a fixed length, we aim to pinpoint the position and type of the break merely by looking at the (unique) sum of the amplitude numbers for small grids.
\end{abstract}

topics: quantum graph, periodic orbit, break in a nanowire grid

\section{Introduction}

In 1992 Gordon, Webb, and Wolpert [1] answered the famous question of Kac: "Can one hear the shape of a drum?" [2], namely are two nonisometric two-dimensional manifolds determined uniquely by their spectra. The answer is no, but these studies gave rise to investigations of spectral problems of not only two-dimensional manifolds but also to one-dimensional manifolds which more simply can be seen as undirected metric graphs.

In 2001 Gutkin and Smilansky [3] and later (in 2005) Kurasov and Nowaczyk [4] studied inverse spectral problems for quantum graphs, i.e., metrised graphs with the Laplace operator defined on the edges and some boundary conditions at the vertices. In both articles the authors used the socalled trace formula to show that it is impossible to determine uniquely the graph from its spectrum. The trace formula connects - in the distributive sense - the spectrum of the operator defined on a graph with metric properties of this graph i.e., with the number of vertices and edges, as well as the total length of the graph and the lengths of all closed paths.

Metrised graphs with the Laplace operator and with matching conditions at the vertices are a good model for free electron motion of conjugated molecules [5], thin superconducting networks [6] and quantum wire circuits [7]. These topics have been studied from a physical and mathematical point of view in many articles, see [8] and the references therein. Also electrical networks, equilateral quantum graphs and quantum lattices have been studied in [9-12] and many others.
Let $G$ be a connected graph with $M$ vertices $v_{m}$ and $N$ edges $e_{n}$ which are intervals on the real line and let $d_{m}$ be the degree of vertex $v_{m}$. We consider the space of square integrable functions on $G$, that is $\mathcal{H} \equiv L^{2}(G)=\oplus_{i=1}^{N} L^{2}\left(e_{i}\right)$. In this paper we will study the Laplace operator defined as $L=\oplus_{i=1}^{N}\left(-\frac{\mathrm{d}^{2}}{\mathrm{~d} x^{2}}\right)$ together with standard (or sometimes called natural or Kirchhoff) matching conditions at the vertices, i.e., at each vertex the values of the functions from all the edges connected at this vertex are the same and the sum of their normal derivatives is equal to zero. These boundary conditions are one of many that define a self-adjoint Laplace operator. Moreover, these conditions can be written in matrix form, where the entries will be called scattering coefficients,

$$
S\left(v_{m}\right)=\left(\sigma_{i j}^{m}\right)=\left(\begin{array}{cccc}
\frac{2-d_{m}}{d_{m}} & \frac{2}{d_{m}} & \cdots & \frac{2}{d_{m}} \\
\frac{2}{d_{m}} & \frac{2-d_{m}}{d_{m}} & \cdots & \frac{2}{d_{m}} \\
\vdots & & \ddots & \vdots \\
\frac{2}{d_{m}} & \frac{2}{d_{m}} & \cdots & \frac{2-d_{m}}{d_{m}}
\end{array}\right) .
$$

The coefficients on the diagonal will be called reflection coefficients $r=\frac{2-d_{m}}{d_{m}}$ and off the diagonal - transition coefficients $t=\frac{2}{d_{m}}$. These numbers (which, when squared are probabilities and hence the rows in the matrix sum to one) represent whether we have a reflection or a transition of the wave function at a certain node in a particular orbit. In other words, if we are on the main diagonal of the matrix, negative numbers indicate that when traversing the orbit on the grid, we are entering and leaving a node by the same edge (reflection). The off-diagonal numbers represent a transition since we enter and leave the node on different edges. 
Another application of scattering coefficients can be found in the recent paper by Baldassari (see [13]) where distribution descriptors for inhomogeneous objects are obtained from the scattering coefficients of the target from the perturbation of reflected waves.

The studies about the uniqueness of the inverse spectral problem in [3] and [4] focus mainly on the lengths of the closed paths called periodic orbits. However, there is more information contained in the trace formula, namely data about all transition and reflection coefficients along periodic orbits of the given geometric length.

We make use of the following notation:

- $P$ is the set of all periodic orbits $p$ in graph $G$;

- $l(p)$ is the geometric length (hereafter length) of periodic orbit $p$;

- $\mathcal{L}=l\left(e_{1}\right)+l\left(e_{2}\right)+\ldots+l\left(e_{N}\right)$ is the total length of graph $G$;

- $\operatorname{prim}(p)$ is the primitive periodic orbit of $p$ (the shortest orbit such that $p$ is a repetition of $\operatorname{prim}(p))$;

- $\left\{k_{j}^{2}\right\}$ is the spectrum of the Laplace operator $L$ on graph $G$ with standard boundary conditions (or any other matching conditions with real entries in the scattering matrices).

The trace formula is then given by

$$
\begin{aligned}
& u(k) \equiv \delta(k)+\sum_{j=1}^{\infty}\left[\delta\left(k-k_{j}\right)+\delta\left(k+k_{j}\right)\right]= \\
& -(N-M+1) \delta(k)+\frac{\mathcal{L}}{\pi} \\
& +\frac{1}{2 \pi} \sum_{p \in P} \mathcal{A}_{p}\left(\mathrm{e}^{\mathrm{i} k l(p)}+\mathrm{e}^{-\mathrm{i} k l(p)}\right),
\end{aligned}
$$

where $\delta(k)$ is the Dirac delta function and the amplitude number $\mathcal{A}_{p}$ consists of the product of all scattering coefficients $\sigma_{i j}^{m}$ along periodic orbit $p$ (the set of all these coefficients will be denoted by $\mathcal{T}(p))$ and the length of primitive orbit $p$. That is

$$
\mathcal{A}_{p}=l(\operatorname{prim}(p))\left(\prod_{\sigma_{i j}^{m} \in \mathcal{T}(p)} \sigma_{i j}^{m}\right) .
$$

Now we focus on a finite $m$ by $n$ grid with rounded corners. In Fig. 1 we indicate with specific symbols the position of the various breaks (not permitted on the rim) which we will be referring to in the latter part of this paper. Note that for a sufficiently large grid some of the symbols occur twice due to symmetry about the diagonal from the corner. Moreover, each corner shows the same pattern of symbols.

Note that any internal node has degree four and any external node (i.e., on the rim) has degree three. The matching conditions are responsible for the transition of the wave function through graph $G$. We assume that there is a break (change of matching conditions) at an internal node in such a way that the node disappears, which leaves the north

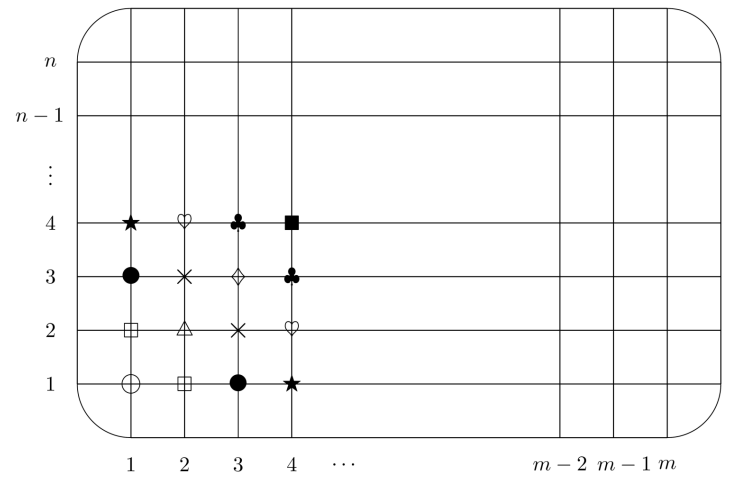

Fig. 1. Diagram of an $m$ by $n$ grid.

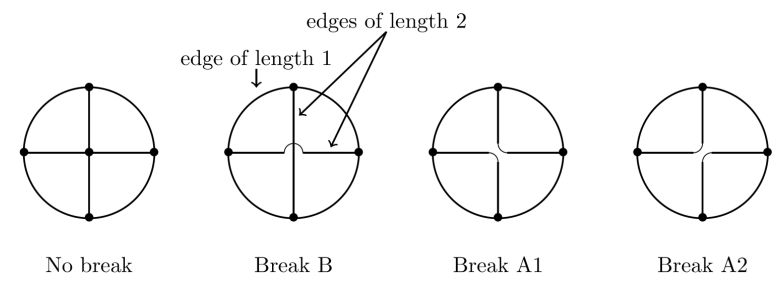

Fig. 2. Types of breaks.

and south components connected by an edge of length two (without a node in the middle), and similarly for the east and west components. This type of break will be called a break of type B. Breaks of type A1 and A2 occur when the connections northsouth and east-west are broken. In addition, for A1 (respectively A2), there are edges of length two connecting the north and east components and the west and south components (respectively north to west and south to east components). For an illustration see Fig. 2. This grid can model a lattice of superconductive nanowires with a break at one of the nodes, where the movement of electrons with low energy can be represented by the Laplace operator. Thus our task will be to identify the place and type of the break by checking the amplitudes of the signal sent into the grid.

Now, we calculate the unique numbers given by

$$
\text { total loss }=T_{b}(k):=\sum_{\substack{p \in P \\ l(p)=k}} \mathcal{A}_{p}-\sum_{\substack{p \in P_{b} \\ l(p)=k}} \mathcal{A}_{p}^{b},
$$

for $k=2,3,4, \ldots$ and $b=\mathrm{B}$ or $\mathrm{A} 1$ or $\mathrm{A} 2$. Here $P_{b}$ is the set of possible paths (periodic orbits) for the grid with a single break at an internal node. Note that it is not necessarily true that $P_{b}$ is a subset of $P$. Also, the set $P_{b}$ will be different for different positions and types of the break.

In practice, we consider $k=2, \ldots, 6$ and find the total loss (or gain) directly by considering which paths are lost and gained when a break occurs in the grid.

By calculating how much the sum of the amplitude numbers over all periodic orbits (all $p$ ) changes for a fixed length $l(p)$, we can locate the position and type of the break merely by looking at these 
unique numbers.

Since we only have vertices of degree 3 and 4 , the scattering matrices we use are

$$
S_{3}=\left(\begin{array}{ccc}
-\frac{1}{3} & \frac{2}{3} & \frac{2}{3} \\
\frac{2}{3} & -\frac{1}{3} & \frac{2}{3} \\
\frac{2}{3} & \frac{2}{3} & -\frac{1}{3}
\end{array}\right)
$$

and

$$
S_{4}=\left(\begin{array}{cccc}
-\frac{1}{2} & \frac{1}{2} & \frac{1}{2} & \frac{1}{2} \\
\frac{1}{2} & -\frac{1}{2} & \frac{1}{2} & \frac{1}{2} \\
\frac{1}{2} & \frac{1}{2} & -\frac{1}{2} & \frac{1}{2} \\
\frac{1}{2} & \frac{1}{2} & \frac{1}{2} & -\frac{1}{2}
\end{array}\right) .
$$

We now illustrate these ideas with an example.

\section{Example}

Suppose we consider all internal orbits (closed paths) of length $l(p)=2$ and $l(p)=4$ on the grid shown in Fig. 2 (No break). We calculate the sum of all amplitude numbers according to (2).

For each $p$ of length two, $l(p)=l(\operatorname{prim}(p))=2)$, we can only use one edge since it must be a closed orbit. There are two possible types of path. For the first type there are four paths from the centre to the rim and back. In other words, there is a reflection at the rim, i.e., a scattering coefficient of $-\frac{1}{3}$ and a reflection at the internal node, i.e., a scattering coefficient of $-\frac{1}{2}$. For the second type there are also four paths on the rim back and forth between two nodes (so here there are two reflections on the rim and thus we have two scattering coefficients of $\left.-\frac{1}{3}\right)$. Since all interactions with nodes are reflections, all the scattering coefficients are negative. Hence we have (we will call this the total amplitude, $\mathcal{A}_{\text {total }}$, for brevity):

$$
\begin{gathered}
\mathcal{A}_{\text {total }}=\sum_{\substack{p \in P \\
l(p)=2}} \mathcal{A}_{p}= \\
2\left[4\left(-\frac{1}{2}\right)\left(-\frac{1}{3}\right)+4\left(-\frac{1}{3}\right)^{2}\right]=\frac{20}{9} .
\end{gathered}
$$

For each $p$ of length four (i.e., $l(p)=4$ ), we can have paths using one, two, or four edges.

(a) One edge: Same possibilities as above but traversed twice (in this case $l(\operatorname{prim}(p))=2$ ). Note that all scattering coefficients from the case $l(p)=2$ are thus squared.

(b) Two edges: Three different types all having $l(p)=l(\operatorname{prim}(p))=4$.

(i) Two edges on the rim (there are four of these). This means that we have two transitions on the rim (each with scattering coefficient $\frac{2}{3}$ ) and two reflections on the rim (each with scattering coefficient $\left.-\frac{1}{3}\right)$. (ii) An interior edge connected to a rim edge. There are eight of these, with one reflection on the rim, one reflection inside and two transitions on the rim. The scattering coefficients are (respectively) $-\frac{1}{3},-\frac{1}{2}$ and $\frac{2}{3}$.

(iii) Two internal edges (of which there are two straight and four bent). This means two internal transitions $\left(\frac{1}{2}\right)$ and two reflections on the $\operatorname{rim}\left(-\frac{1}{3}\right)$.

(c) Four edges: All four edges on the rim or two edges on the rim and the other two from the rim to the centre. In both cases we only have transitions. For the first case all the scattering coefficients are $\frac{2}{3}$, but in the latter case one of them is $\frac{1}{2}$ and the other three are $\frac{2}{3}$. Here $l(p)=l(\underset{\operatorname{prim}}{\operatorname{mom}}(p))=4$.

Thus the total amplitude is

$$
\begin{aligned}
& \mathcal{A}_{\text {total }}=\sum_{\substack{p \in P \\
l(p)=4}} \mathcal{A}_{p}= \\
& 2\left[4\left(-\frac{1}{2}\right)^{2}\left(-\frac{1}{3}\right)^{2}+4\left(-\frac{1}{3}\right)^{4}\right] \\
& +4\left[4\left(-\frac{1}{3}\right)^{2}\left(\frac{2}{3}\right)^{2}+8\left(-\frac{1}{2}\right)\left(-\frac{1}{3}\right)\left(\frac{2}{3}\right)^{2}\right. \\
& \left.+6\left(\frac{1}{2}\right)^{2}\left(-\frac{1}{3}\right)^{2}+\left(\frac{2}{3}\right)^{4}+4\left(\frac{1}{2}\right)\left(\frac{2}{3}\right)^{3}\right]=\frac{592}{81} .
\end{aligned}
$$

We now compare these answers to the sum of amplitude numbers obtained from the grid with break B (see Fig. 2).

For $l(p)=2$, if we have break B as indicated, we will no longer have the paths which go between the centre and the rim. Thus the only remaining closed orbits of length two are those using an edge on the rim. This yields a resulting total amplitude $\mathcal{A}_{p}^{B}$ (when there is a break of type B) given by

$$
\mathcal{A}_{\text {total }}^{\mathrm{B}}=\sum_{\substack{p \in P_{B} \\ l(p)=2}} \mathcal{A}_{p}^{\mathrm{B}}=2\left[4\left(-\frac{1}{3}\right)^{2}\right]=\frac{8}{9} .
$$

Note that this total is the same for breaks A1 and A2, i.e., $\mathcal{A}_{\text {total }}^{\mathrm{B}}=\mathcal{A}_{\text {total }}^{\mathrm{A} 1}=\mathcal{A}_{\text {total }}^{\mathrm{A} 2}$.

For paths of length four, we lose all paths that would reflect at or transit through the centre node. This means that the only paths that remain are those that use only edges on the rim (either one, two, or four). However, we gain two new types of paths which we describe below.

- The first type is the path traversing from north to south and back and the path from east to west and back (since the north-south and east-west edges are now of length two with no node in the middle). These paths both have two reflections on the rim, each with scattering coefficient $-\frac{1}{3}$. 
- The second type is the paths traversing the semicircles comprised of an interior edge (of length two with no node in the middle) and two edges on the rim. There are four such paths and they have three transitions on the rim, each with scattering coefficient $\frac{2}{3}$.

Thus

$$
\begin{gathered}
\mathcal{A}_{\text {total }}^{\mathrm{B}}=\sum_{\substack{p \in P_{B} \\
l(p)=4}} \mathcal{A}_{p}^{\mathrm{B}}=2\left[4\left(-\frac{1}{3}\right)^{4}\right] \\
+4\left[4\left(-\frac{1}{3}\right)^{2}\left(\frac{2}{3}\right)^{2}+\left(\frac{2}{3}\right)^{4}\right. \\
+\underbrace{\left.2\left(-\frac{1}{3}\right)^{2}+4\left(\frac{2}{3}\right)^{3}\right]}_{\text {gain }}=\frac{592}{81} .
\end{gathered}
$$

For breaks $\mathrm{A} 1$ and $\mathrm{A} 2, \mathcal{A}_{\mathrm{total}}^{\mathrm{A} 1}=\mathcal{A}_{\mathrm{total}}^{\mathrm{A} 2}=\frac{136}{81}$ since $\mathcal{A}_{\text {total }}$ is the same and we lose exactly the same paths as for break B. However, we do not gain any new paths and hence the two underbraced terms above will not exist for breaks A1 and A2.

In general, for a larger grid, the position of the break can vary and so to identify the position (and type) of the break we compare the sums of $\mathcal{A}_{p}$ to $\mathcal{A}_{p}^{b}$, where $b$ denotes the type of break, i.e., $\mathrm{B}, \mathrm{A} 1$, or A2. This will be done for various values of $l(p)$. In the next section we provide the details.

\section{Orbits of finite length}

The unique numbers given by ( 3 ) for $k=2, \ldots, 6$ and $b=\mathrm{B}$ or $\mathrm{A} 1$ or $\mathrm{A} 2$ are calculated in this section. Note that when we refer to a break position, we are referring to the south-west corner of the grid (see Fig. 1). By symmetry, all the computations below also hold for the north-east corner of the grid. The only changes are in the north-west and south east corners of the grid where the roles of breaks A1 and A2 are interchanged.

\subsection{Orbits of length two, three and five for break B}

In this section we focus on paths with length $l(p)=2, l(p)=3$ and $l(p)=5$ for break B. In these cases $l(\operatorname{prim}(p))=l(p)$. These paths are comparatively easy as will be seen below.

There are only three break positions which have distinct $T_{\mathrm{B}}(2)$ values (see $(3)$ ), namely the breaks in positions $\bigcirc, \square$ and $\triangle$ in Fig. 1. Recall that there are two different positions (indicated by $\square$ ) which will have the same $T_{\mathrm{B}}(2)$ values due to symmetry. The remaining break positions yield the same $T_{\mathrm{B}}(2)$ values as the $\square$ (for the outermost breaks) and the $\triangle$ (for the rest). This is under the assumption that the grid is at least three-by-three $(m, n \geq 3)$. In this case the initial number outside the bracket is $l(\operatorname{prim}(p))=l(p)=2$.
Firstly, for $\bigcirc$, we calculate $T_{\mathrm{B}}(2)$ as follows:

$$
T_{\mathrm{B}}(2)=2[2 \underbrace{\left(-\frac{1}{2}\right)\left(-\frac{1}{3}\right)}_{\text {reach the rim }}+2 \underbrace{\left(-\frac{1}{2}\right)^{2}}_{\text {internal }}]=\frac{5}{3} .
$$

There are only four paths of length two (which are affected by a break at $\bigcirc$ ): two which reach the rim and two which are internal. All the scattering coefficients are negative since they are reflections.

For the break at $\square$ we again have four paths which are affected, but three are internal and only one reaches the rim, giving

$$
T_{\mathrm{B}}(2)=2\left[\left(-\frac{1}{2}\right)\left(-\frac{1}{3}\right)+3\left(-\frac{1}{2}\right)^{2}\right]=\frac{11}{6} .
$$

Finally, the break at $\triangle$ has that all four affected paths are internal. Hence

$$
T_{\mathrm{B}}(2)=2\left[4\left(-\frac{1}{2}\right)^{2}\right]=2 .
$$

The above calculations demonstrate that all the other breaks (whose positions are indicated in Fig. 1) would have a total loss of either $\frac{11}{6}$ or 2 .

Observe that the total loss is non-decreasing as the break position moves further from the corner.

Now we consider paths of length three. This is a very special case since we can only obtain such closed paths on a corner. In other words, only break $\bigcirc$ is applicable and the (single) path consists only of transitions, two on the rim and one inside. In this case the number outside the bracket is $l(\operatorname{prim}(p))=$ $l(p)=3$. This results in

$$
T_{\mathrm{B}}(3)=3\left[\left(\frac{1}{2}\right)\left(\frac{2}{3}\right)^{2}\right]=\frac{2}{3} .
$$

Paths of length five are similar in that they also need to be at a corner. For this reason we need only consider the breaks at $\bigcirc$ and $\square$. As with paths of length two, the grid must be at least three-by-three for the results in Table I to be valid.

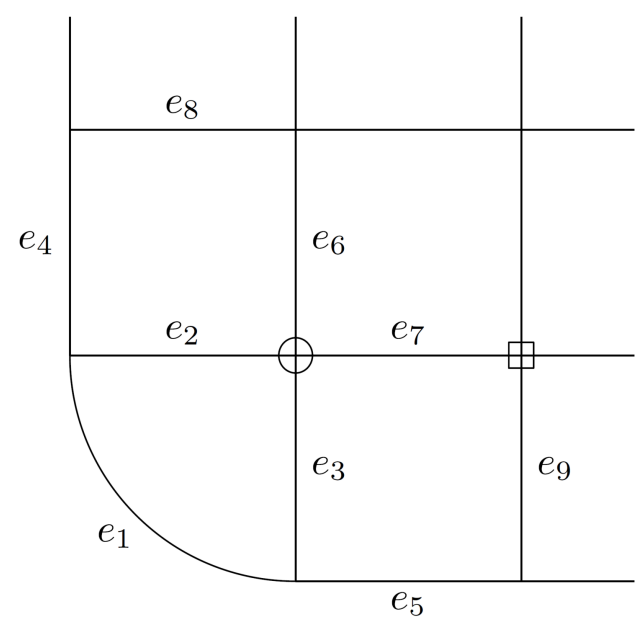

Fig. 3. Diagram showing edges used in paths of length five. 
Total loss: $l(p)=5$ for break B at $\bigcirc$.

\begin{tabular}{c|c|c|c|c}
\hline \hline \# of edges & Repeated edge & Scattering coefficients & Loss & Gain \\
\hline 3 & $e_{1}$ & $\left(\frac{1}{2}\right)\left(\frac{2}{3}\right)^{2}\left(-\frac{1}{3}\right)^{2}$ & $5 \times \frac{2}{81}$ & - \\
3 & $e_{2}$ or $e_{3}$ & $\left(\frac{1}{2}\right)\left(\frac{2}{3}\right)^{2}\left(-\frac{1}{3}\right)\left(-\frac{1}{2}\right)$ & $5 \times 2\left(\frac{1}{27}\right)$ & - \\
4 & $e_{4}$ or $e_{5}$ & $\left(\frac{1}{2}\right)\left(\frac{2}{3}\right)^{3}\left(-\frac{1}{3}\right)$ & $5 \times 2\left(-\frac{4}{81}\right)$ & - \\
4 & $e_{6}$ or $e_{7}$ & $\left(\frac{1}{2}\right)^{2}\left(\frac{2}{3}\right)^{2}\left(-\frac{1}{2}\right)$ & $5 \times 2\left(-\frac{1}{18}\right)$ & - \\
5 & none & $\left(\frac{1}{2}\right)^{2}\left(\frac{2}{3}\right)^{3}$ & $5 \times 2\left(\frac{2}{27}\right)$ & $5 \times 2\left(\frac{4}{27}\right)$ \\
\hline $4^{a}$ & none & $\left(\frac{1}{2}\right)\left(\frac{2}{3}\right)^{3}$ & - & \\
\hline
\end{tabular}

${ }^{a}$ One edge of length two and three edges of length one.

For the break at $\bigcirc$, we will firstly make use of the cycle comprised of edges $e_{1}, e_{2}$ and $e_{3}$ (see Fig. 3 ). In order to make paths of length five, we also need to include one more edge that is traversed twice. This could be any of $e_{1}$ to $e_{7}$ resulting in a path using either three or four edges. Secondly, we consider the paths with only transitions, namely traversing the edges $e_{1}, e_{4}, e_{8}, e_{6}, e_{3}$ or $e_{1}, e_{2}, e_{7}, e_{9}, e_{5}$. We also gain new paths with only transitions, in particular $e_{1}, e_{4}, e_{8}, e_{6 ; 3}$ and $e_{1}, e_{2 ; 7}, e_{9}, e_{5}$ where $e_{6 ; 3}$ (respectively $\left.e_{2 ; 7}\right)$ is the edge of length two made up of edges $e_{6}$ and $e_{3}$ (respectively $e_{2}$ and $e_{7}$ ) with no node in the middle. Table I shows the details. Note that the total loss in this case is negative, indicating a positive total gain.

The other break relevant to this path length is at $\square$. There are two possible paths. The first makes use of one edge $\left(e_{7}\right.$, traversed twice) and the cycle comprised of edges $e_{1}, e_{2}$ and $e_{3}$ (see Fig. 3). The second possible path is of length five with only transitions, namely $e_{1}, e_{2}, e_{7}, e_{9}, e_{5}$. In this case we gain no new paths, thus for the break at $\square$ we have a total loss of

$$
\begin{aligned}
& T_{\mathrm{B}}(5)= \\
& 5[\underbrace{\left(\frac{1}{2}\right)^{2}\left(\frac{2}{3}\right)^{2}\left(-\frac{1}{2}\right)}_{e_{7} \text { traversed twice }}+\underbrace{\left(\frac{1}{2}\right)^{2}\left(\frac{2}{3}\right)^{3}}_{\text {only transitions }}]=\frac{5}{54} .
\end{aligned}
$$

\subsection{Orbits of length two, three and five for breaks A1 and A2}

For paths with length $l(p)=2$ and breaks A1 or A2 (see Fig. 2) the calculations for $T_{\mathrm{A} 1}(2)$ and $T_{\mathrm{A} 2}(2)$ are identical to those for break $\mathrm{B}$ (see previous section). That means if the break occurs at $\bigcirc$ then $T_{\mathrm{A} 1}(2)=T_{\mathrm{A} 2}(2)=\frac{5}{3}$. If it occurs at $\square$ then $T_{\mathrm{A} 1}(2)=T_{\mathrm{A} 2}(2)=\frac{11}{6}$ and at $\triangle$ the value of $T_{\mathrm{A} 1}(2)=T_{\mathrm{A} 2}(2)=2$.

Now we consider paths of length three (where the only possible paths are on a corner), i.e., only break $\bigcirc$ needs to be considered. If the break at $\bigcirc$ is type A2 the calculations mimic those for break B giving $T_{\mathrm{A} 2}(3)=\frac{2}{3}$. However, for break type A1 at $\bigcirc$ although we lose the term corresponding to the path with three transitions, we gain a new term corresponding to a path with only two transitions on the rim. Thus $T_{\mathrm{A} 1}(3)=\frac{2}{3}-\frac{4}{3}=-\frac{2}{3}$, which means that the total loss is actually a gain and we can distinguish between breaks B and A1.

Recall that paths of length five also need to be at a corner. Therefore, we consider only the breaks at $\bigcirc$ and $\square$. Note that the loss (for both breaks A1 or A2) is the same as for break B.

In Table II we show the details for breaks A1 and $\mathrm{A} 2$ at $\bigcirc$. The only changes in comparison to break B occur in the gain and thus only for A1. For break A2 there will be no gain at position $\bigcirc$.

Let us now consider break A1 or A2 at $\square$. There are two possible paths. The first makes use of one edge $\left(e_{7}\right.$, traversed twice) and the cycle comprised of edges $e_{1}, e_{2}$ and $e_{3}$ (see Fig. 3). The second possible path of length five has only transitions and uses edges $e_{1}, e_{2}, e_{7}, e_{9}, e_{5}$. For the A2 break at $\square$ the calculations for the total loss are the same as for break B (since we have no gains), i.e., $T_{\mathrm{A} 2}(5)=\frac{5}{54}$. For break A1 we gain a new path yielding a total loss of

$$
T_{\mathrm{A} 1}(5)=5[\underbrace{\left(\frac{1}{54}\right)}_{\text {see }(4)}-\underbrace{\left(\frac{2}{3}\right)^{3}\left(\frac{1}{2}\right)}_{\text {gain }}]=-\frac{35}{54} .
$$

\subsection{Orbits of length four for break B}

In the case of paths with length $l(p)=4$, we consider breaks $\bigcirc, \square, \triangle, \bigcirc, \times$ and $\diamond$ (see Fig. 1). These are the columns in Table III. Note that we require at least five rows and at least five columns in our grid for the results in Table III to hold.

Since there are many different possible paths, we now provide a suitable code describing each type of path, according to (in the given order):

- the total number of edges on the grid with no break,

- the number of nodes on the rim,

- the number of edges on the rim. 
Total loss: $l(p)=5$ for break $\mathrm{A} 1$ and $\mathrm{A} 2$ at $\bigcirc$.

TABLE II

\begin{tabular}{c|c|c|c|c}
\hline \hline \# of edges & Repeated edge & Scattering coefficients & Loss A1 \& A2 & Gain A1 \\
\hline 3 & $e_{1}$ & $\left(\frac{1}{2}\right)\left(\frac{2}{3}\right)^{2}\left(-\frac{1}{3}\right)^{2}$ & $5 \times \frac{2}{81}$ & - \\
$2^{a}$ & $e_{1}$ & $\left(\frac{2}{3}\right)^{2}\left(-\frac{1}{3}\right)^{2}$ & - & $5 \times \frac{4}{81}$ \\
3 & $e_{2}$ or $e_{3}$ & $\left(\frac{1}{2}\right)\left(\frac{2}{3}\right)^{2}\left(-\frac{1}{3}\right)\left(-\frac{1}{2}\right)$ & $5 \times 2\left(\frac{1}{27}\right)$ & - \\
4 & $e_{4}$ or $e_{5}$ & $\left(\frac{1}{2}\right)\left(\frac{2}{3}\right)^{3}\left(-\frac{1}{3}\right)$ & $5 \times 2\left(-\frac{4}{81}\right)$ & $5 \times 2\left(-\frac{8}{81}\right)$ \\
$3^{b}$ & $e_{4}$ or $e_{5}$ & $\left(\frac{2}{3}\right)^{3}\left(-\frac{1}{3}\right)$ & - & - \\
4 & $e_{6}$ or $e_{7}$ & $\left(\frac{1}{2}\right)^{2}\left(\frac{2}{3}\right)^{2}\left(-\frac{1}{2}\right)$ & $5 \times 2\left(-\frac{1}{18}\right)$ & - \\
5 & none & $\left(\frac{1}{2}\right)^{2}\left(\frac{2}{3}\right)^{3}$ & $\frac{25}{27}$ & \\
\hline$T_{\mathrm{A} 1}(5)$ & & & $\frac{5}{27}$ & \\
$T_{\mathrm{A} 2}(5)$ & \multicolumn{3}{c}{} \\
\hline
\end{tabular}

${ }^{a}$ One edge of length two and one edge of length one.

${ }^{b}$ One edge of length two and two edges of length one.

Total loss $l(p)=4$ at the six positions for break B.

TABLE III

\begin{tabular}{|c|c|c|c|c|c|c|}
\hline & $\bigcirc$ & $\square$ & 0 & $\triangle$ & $x$ & $\diamond$ \\
\hline 110 & $\frac{1}{9}$ & $\frac{1}{18}$ & $\frac{1}{18}$ & 0 & 0 & 0 \\
\hline 100 & $\frac{1}{4}$ & $\frac{3}{8}$ & $\frac{3}{8}$ & $\frac{1}{2}$ & $\frac{1}{2}$ & $\frac{1}{2}$ \\
\hline 221 & $\frac{32}{27}$ & $\frac{16}{27}$ & $\frac{16}{27}$ & 0 & 0 & 0 \\
\hline 220 & $\frac{1}{9}$ & 0 & 0 & 0 & 0 & 0 \\
\hline 210 loss & 1 & 1 & $\frac{5}{6}$ & $\frac{1}{3}$ & $\frac{1}{6}$ & 0 \\
\hline 210 gain & $\frac{4}{3}$ & $\frac{2}{3}$ & $\frac{2}{3}$ & 0 & 0 & 0 \\
\hline 200 loss & $\frac{5}{4}$ & $\frac{9}{4}$ & $\frac{5}{2}$ & 4 & $\frac{17}{4}$ & $\frac{9}{2}$ \\
\hline 200 gain & 0 & 1 & 1 & 2 & 2 & 2 \\
\hline 421 & $\frac{8}{9}$ & $\frac{8}{9}$ & $\frac{8}{9}$ & 0 & 0 & 0 \\
\hline 400 & $\frac{1}{4}$ & $\frac{1}{2}$ & $\frac{1}{2}$ & 1 & 1 & 1 \\
\hline$T_{\mathrm{B}}(4)$ & 3.712963 & 3.995370 & 4.078704 & 3.833333 & 3.916667 & 4.000000 \\
\hline
\end{tabular}

It is understood that for new paths gained once a break occurs, the total number of edges used to make a path will be one less since the break creates a new edge of length two with no node in the middle (see Fig. 2). For example, a path with code 221 will have two edges in total, two nodes on the rim and one edge on the rim. There are three nodes in total on this path and the second edge goes from the rim to the third (interior) node. For the new paths gained, with code 200 say, the 2 corresponds to two edges on the grid with no break. On the grid with a break it is the one edge with length two made up of the two original edges with no node in the middle.

Note that for the paths using only one edge $l(\operatorname{prim}(p))=2$ and for all the remaining closed paths of length 4 , we have $l(\operatorname{prim}(p))=4$. Thus, the total loss in Table III is obtained by multiplying the scattering coefficients by the corresponding $l(\operatorname{prim}(p))$ and summing.

It is interesting to note that the total loss increases, the further one moves away from the corner on the main diagonal. Since the total loss values for the different breaks are all unique (subject to symmetry about the diagonal), we are able to identify the position of the break.

\subsection{Orbits of length four for breaks A1 and A2}

In this subsection we consider paths with length $l(p)=4$ and breaks $\mathrm{A} 1$ and $\mathrm{A} 2$ at $\bigcirc, \square, \triangle$, $\times$ and $\diamond$. The only case where $T_{\mathrm{A} 1}(4) \neq T_{\mathrm{A} 2}(4)$ is when the break is at $\bigcirc$ and thus we treat this case separately. For the remaining cases see Table IV.

Note that all the losses are identical to those corresponding to break $\mathrm{B}$. Hence the only differences occur in the gains.

For break $\mathrm{A} 1$ at $\bigcirc$ the gains occur at paths with codes 200,220, and 400 given respectively by $1, \frac{4}{9}$, and $\frac{1}{2}$. Thus,

$$
T_{\mathrm{A} 1}(4)=\frac{545}{108}-\left(1+\frac{4}{9}+\frac{1}{2}\right)=3.101852 .
$$

Similarly for break A2 at $\bigcirc$ the gains now occur at paths with codes 210 and 421 given respectively by $\frac{4}{3}$ and $\frac{16}{9}$. Therefore,

$$
T_{\mathrm{A} 2}(4)=\frac{545}{108}-\left(\frac{4}{3}+\frac{16}{9}\right)=1.935185 .
$$

Note that $\frac{545}{108}=T_{\mathrm{B}}(4)+\frac{4}{3}$ (i.e., the total loss from break $\mathrm{B}$ at $\bigcirc$ plus the gains from break $\mathrm{B}$ at $\bigcirc$ ), see Table III. 
Total loss: $l(p)=4$ at the five break positions for breaks A1 and A2.

TABLE IV

\begin{tabular}{c|c|c|c|c|c}
\hline \hline & $\square$ & $\bullet$ & $\triangle$ & $\times$ & $\diamond$ \\
\hline 110 & $\frac{1}{18}$ & $\frac{1}{18}$ & 0 & 0 & 0 \\
100 & $\frac{3}{8}$ & $\frac{3}{8}$ & $\frac{1}{2}$ & $\frac{1}{2}$ & $\frac{1}{2}$ \\
221 & $\frac{16}{27}$ & $\frac{16}{27}$ & 0 & 0 & 0 \\
210 loss & 1 & $\frac{5}{6}$ & $\frac{1}{3}$ & $\frac{1}{6}$ & 0 \\
210 gain & $\frac{2}{3}$ & $\frac{2}{3}$ & 0 & 0 & $\frac{9}{2}$ \\
200 loss & $\frac{9}{2}$ & 1 & 4 & $\frac{17}{4}$ & 2 \\
200 gain & 1 & $\frac{8}{9}$ & 0 & 0 & 0 \\
421 loss & $\frac{8}{9}$ & $\frac{8}{2}$ & 0 & 0 & 1 \\
421 gain & $\frac{8}{9}$ & $\frac{1}{2}$ & 1 & 1 & 1 \\
400 loss & $\frac{1}{2}$ & 2.689815 & 2.833333 & 2.916667 & 3.000000 \\
\hline
\end{tabular}

Total loss: $l(p)=6$ at six positions for break B.

TABLE V

\begin{tabular}{|c|c|c|c|c|c|c|}
\hline & 0 & $\square$ & - & $\triangle$ & $\times$ & $\diamond$ \\
\hline 110 & $\frac{1}{54}$ & $\frac{1}{108}$ & $\frac{1}{108}$ & 0 & 0 & 0 \\
\hline 100 & $\frac{1}{16}$ & $\frac{3}{32}$ & $\frac{3}{32}$ & $\frac{1}{8}$ & $\frac{1}{8}$ & $\frac{1}{8}$ \\
\hline 221 & $\frac{8}{27}+\frac{16}{81}$ & $\frac{4}{27}+\frac{8}{81}$ & $\frac{8}{81}+\frac{4}{27}$ & 0 & 0 & 0 \\
\hline 220 & $\frac{1}{18}$ & 0 & 0 & 0 & 0 & 0 \\
\hline 210 & $\frac{1}{4}+\frac{3}{8}$ & $\frac{3}{8}+\frac{1}{4}$ & $\frac{5}{16}+\frac{5}{24}$ & $\frac{1}{8}+\frac{1}{12}$ & $\frac{1}{16}+\frac{1}{24}$ & 0 \\
\hline 200 & $\frac{15}{16}$ & $\frac{27}{16}$ & $\frac{15}{8}$ & 3 & $\frac{51}{16}$ & $\frac{27}{8}$ \\
\hline 332 & $\frac{64}{81}-\frac{16}{81}$ & $\frac{32}{81}-\frac{8}{81}$ & $\frac{32}{81}-\frac{8}{81}$ & 0 & 0 & 0 \\
\hline 331 & $\frac{4}{27}$ & 0 & 0 & 0 & 0 & 0 \\
\hline 321 loss & $\frac{4}{27}+\frac{8}{9}+\frac{4}{3}+\frac{4}{27}$ & $\frac{4}{3}+\frac{16}{27}$ & $\frac{10}{9}+\frac{16}{27}$ & $\frac{4}{9}$ & $\frac{2}{9}$ & 0 \\
\hline 321 gain & $\frac{16}{9}$ & $\frac{8}{9}$ & $\frac{8}{9}$ & 0 & 0 & 0 \\
\hline 320 loss & $\frac{1}{6}-\frac{1}{12}$ & $\frac{1}{8}-\frac{1}{24}$ & $\frac{1}{12}$ & 0 & 0 & 0 \\
\hline 320 gain & $\frac{1}{3}$ & 0 & 0 & 0 & 0 & 0 \\
\hline 310 loss & $\frac{3}{4}-\frac{3}{8}$ & $\frac{15}{16}-\frac{7}{16}$ & $\frac{7}{8}-\frac{7}{16}$ & $\frac{7}{8}-\frac{1}{4}$ & $\frac{1}{2}-\frac{1}{8}$ & $\frac{1}{8}$ \\
\hline 310 gain & 1 & $\frac{3}{2}$ & $\frac{5}{4}$ & $\frac{1}{2}$ & $\frac{1}{4}$ & 0 \\
\hline 300 loss & $\frac{21}{16}-\frac{3}{16}$ & $\frac{81}{32}-\frac{15}{32}$ & $3-\frac{9}{16}$ & $\frac{39}{8}-\frac{3}{2}$ & $\frac{183}{32}-\frac{21}{16}$ & $\frac{105}{16}-\frac{3}{2}$ \\
\hline 300 gain & 0 & $\frac{9}{8}$ & $\frac{3}{2}$ & $\frac{15}{4}$ & $\frac{33}{8}$ & $\frac{9}{2}$ \\
\hline 421 & $\frac{4}{27}+\frac{1}{3}+\frac{4}{9}$ & $\frac{4}{27}+\frac{1}{3}+\frac{4}{9}$ & $\frac{4}{27}+\frac{1}{3}+\frac{4}{9}$ & 0 & 0 & 0 \\
\hline 400 & $\frac{3}{8}$ & $\frac{3}{4}$ & $\frac{3}{4}$ & $\frac{3}{2}$ & $\frac{3}{2}$ & $\frac{3}{2}$ \\
\hline 532 & $-\frac{16}{27}$ & $-\frac{16}{27}$ & $-\frac{16}{27}$ & 0 & 0 & 0 \\
\hline 531 & $-\frac{2}{9}$ & $-\frac{1}{9}$ & 0 & 0 & 0 & 0 \\
\hline 521 & $-\frac{4}{3}$ & $-\frac{3}{2}$ & $-\frac{5}{3}$ & $-\frac{2}{3}$ & $-\frac{1}{3}$ & 0 \\
\hline 510 & $-\frac{1}{4}$ & $-\frac{3}{8}$ & $-\frac{1}{4}$ & $-\frac{1}{2}$ & $-\frac{1}{4}$ & 0 \\
\hline 500 & $-\frac{9}{16}$ & $-\frac{39}{32}$ & $-\frac{3}{2}$ & $-\frac{21}{8}$ & $-\frac{51}{16}$ & $-\frac{15}{4}$ \\
\hline 632 loss & $\frac{4}{9}$ & $\frac{4}{9}$ & $\frac{2}{3}$ & 0 & 0 & 0 \\
\hline 632 gain & 0 & $\frac{4}{9}$ & $\frac{4}{9}$ & 0 & 0 & 0 \\
\hline 621 loss & $\frac{1}{3}$ & $\frac{1}{2}$ & $\frac{1}{3}$ & $\frac{2}{3}$ & $\frac{1}{3}$ & 0 \\
\hline 621 gain & $\frac{2}{3}$ & $\frac{2}{3}$ & $\frac{2}{3}$ & 0 & 0 & 0 \\
\hline 600 loss & $\frac{3}{16}$ & $\frac{3}{8}$ & $\frac{15}{32}$ & $\frac{3}{4}$ & $\frac{15}{16}$ & $\frac{9}{8}$ \\
\hline 600 gain & 0 & $\frac{3}{16}$ & $\frac{3}{16}$ & $\frac{3}{4}$ & $\frac{3}{4}$ & $\frac{3}{4}$ \\
\hline Total loss & 2.563272 & 1.915895 & 1.902006 & 1.902778 & 2.295139 & 2.312500 \\
\hline
\end{tabular}


3.5. Orbits of length six for break B

In this case, our focus is on paths with length $l(p)=6$. Note that since the path is longer there are also more break positions (namely $\star, \varnothing, \boldsymbol{k}$, and which give us a unique value of $\mathcal{A}_{\text {total }}^{b}$. These are shown in Fig. 1. This also implies that the values in Tables V and VI will only be valid if our minimum grid size is seven rows and seven columns.

For paths using only one edge $l(\operatorname{prim}(p))=2$. For the path on the corner traversing $e_{1}, e_{2}, e_{3}$ twice (see Fig. 3) with only transition coefficients, $l(\operatorname{prim}(p))=3$. This particular case is only applicable when a break occurs at $\bigcirc$. In all other cases $l(p)=l(\operatorname{prim}(p))=6$.

TABLE VI

Total loss: $l(p)=6$ at the four extra positions for break B.

\begin{tabular}{|c|c|c|c|c|}
\hline & $\star$ & 8 & \& & $\mathbf{\square}$ \\
\hline 110 & $\frac{1}{108}$ & 0 & 0 & 0 \\
\hline 100 & $\frac{3}{32}$ & $\frac{1}{8}$ & $\frac{1}{8}$ & $\frac{1}{8}$ \\
\hline 221 & $\frac{4}{27}+\frac{8}{81}$ & 0 & 0 & 0 \\
\hline 220 & 0 & 0 & 0 & 0 \\
\hline 210 & $\frac{5}{16}+\frac{5}{24}$ & $\frac{1}{16}+\frac{1}{24}$ & 0 & 0 \\
\hline 200 & $\frac{15}{8}$ & $\frac{51}{16}$ & $\frac{27}{8}$ & $\frac{27}{8}$ \\
\hline 332 & $\frac{32}{81}-\frac{8}{81}$ & 0 & 0 & 0 \\
\hline 331 & 0 & 0 & 0 & 0 \\
\hline 321 loss & $\frac{10}{9}+\frac{16}{27}$ & $\frac{2}{9}$ & 0 & 0 \\
\hline 321 gain & $\frac{8}{9}$ & 0 & 0 & 0 \\
\hline 320 loss & $\frac{1}{12}$ & 0 & 0 & 0 \\
\hline 320 gain & 0 & 0 & 0 & 0 \\
\hline 310 loss & $\frac{13}{16}-\frac{7}{16}$ & $\frac{7}{16}$ & $\frac{1}{16}$ & 0 \\
\hline 310 gain & $\frac{5}{4}$ & $\frac{1}{4}$ & 0 & 0 \\
\hline 300 loss & $\frac{99}{32}-\frac{9}{16}$ & $\frac{93}{16}-\frac{21}{16}$ & $\frac{213}{32}-\frac{3}{2}$ & $\frac{27}{4}-\frac{3}{2}$ \\
\hline 300 gain & $\frac{3}{2}$ & $\frac{33}{8}$ & $\frac{9}{2}$ & $\frac{9}{2}$ \\
\hline 421 & $\frac{4}{27}+\frac{1}{3}+\frac{4}{9}$ & 0 & 0 & 0 \\
\hline 400 & $\frac{3}{4}$ & $\frac{3}{2}$ & $\frac{3}{2}$ & $\frac{3}{2}$ \\
\hline 532 & $-\frac{16}{27}$ & 0 & 0 & 0 \\
\hline 531 & 0 & 0 & 0 & 0 \\
\hline 521 & $-\frac{5}{3}$ & $-\frac{1}{3}$ & 0 & 0 \\
\hline 510 & $-\frac{1}{4}$ & $-\frac{1}{4}$ & 0 & 0 \\
\hline 500 & $-\frac{3}{2}$ & $-\frac{51}{16}$ & $-\frac{15}{4}$ & $-\frac{15}{4}$ \\
\hline 632 loss & $\frac{2}{3}$ & 0 & 0 & 0 \\
\hline 632 gain & $\frac{4}{9}$ & 0 & 0 & 0 \\
\hline 621 loss & $\frac{1}{3}$ & $\frac{1}{3}$ & 0 & 0 \\
\hline 621 gain & $\frac{2}{3}$ & 0 & 0 & 0 \\
\hline 600 loss & $\frac{15}{32}$ & $\frac{15}{16}$ & $\frac{9}{8}$ & $\frac{9}{8}$ \\
\hline 600 gain & $\frac{3}{16}$ & $\frac{3}{4}$ & $\frac{3}{4}$ & $\frac{3}{4}$ \\
\hline Total loss & 1.933256 & 2.451389 & 2.343750 & 2.375000 \\
\hline
\end{tabular}

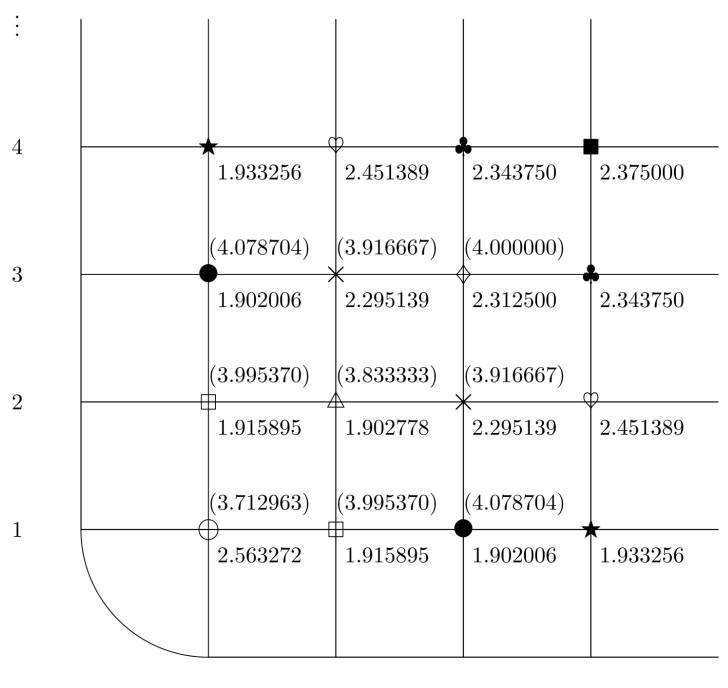

Fig. 4. Summary of total loss for $l(p)=4$ (in brackets) and $l(p)=6$ (no brackets) for break B.

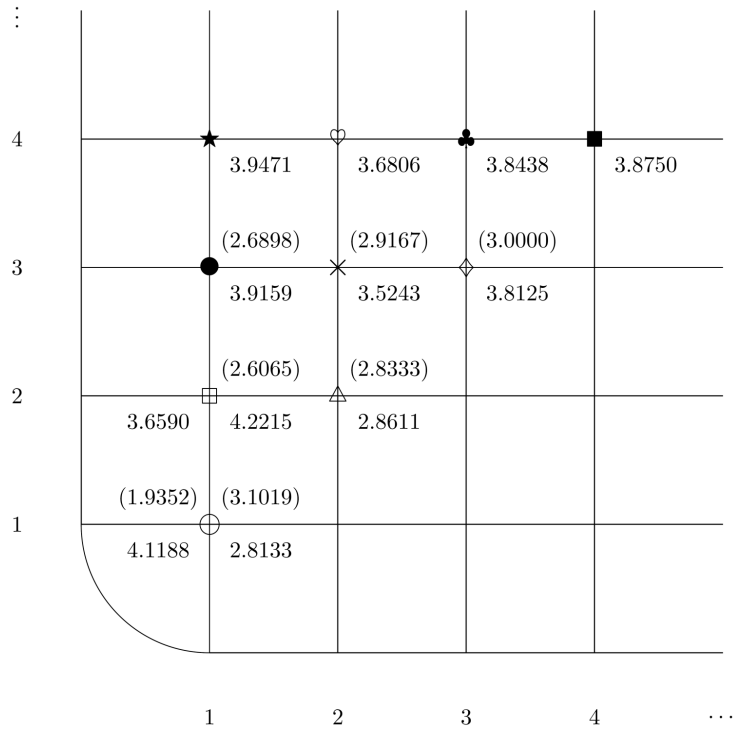

Fig. 5. As in Fig. 4, but for breaks A1 and A2.

Since all of this information will not easily fit into one table, we will have two tables, with the new break positions $\star, \nabla, \boldsymbol{\beta}$, and $\mathbf{\square}$ in the second table. These four break positions cannot be identified uniquely for $l(p)<6$.

In Fig. 4 we condense the results for break B for paths of length four and six. The values in round brackets north-east of the break are for $l(p)=4$. The values south-east of the break correspond to $l(p)=6$.

\subsection{Orbits of length six for breaks A1 and A2}

As can be seen from Sect. 3.5 the calculations for orbits of length 6 are very cumbersome as such we will omit the details and provide a summary of the final results depicted in Fig. 5. 
For comparison purposes, we have included the values for $l(p)=4$ in round brackets northeast of the break. In addition, for $l(p)=4$, only $\bigcirc$ has different total loss values for break A1 and A2, and hence we put the value for A2 north-west of the break. The values south-east of the break correspond to $l(p)=6$, except for the two cases where the total loss for break A1 is different to the total loss for break A2 $(\bigcirc$ and $\square)$. In these cases we put the total loss for A2 south-west of the break in the diagram.

\section{Conclusion}

The following inverse problem has been solved: Given the amplitudes of the signal sent into a quantum grid it is possible to identify if there is a break at an internal node in the grid and if so where the break occurs and what type of break it is. Up to symmetry we have shown that this identification is unique for quantum grids of size $7 \times 7$ or smaller. If the grid is bigger than $7 \times 7$ then the results in the paper are still applicable, in particular for $l(p)=6$ if the break occurs within four rows or columns from the rim then its position and type can be uniquely classified. However, if the break occurs deeper into the grid at position $(i, j)$, say, for $j>4$ and $i=1$ (respectively 2,3 , or $>3$ ) we would have the same amplitude signal as when the break occurs at $\star$ (respectively $\varnothing$, $\mathbf{s}$, or $\mathbf{\square})$. Similarly if the roles of $i$ and $j$ are reversed (by symmetry).

Uniqueness could be extended to larger grids (more break positions) by using longer periodic orbits, however an alternative approach should be considered as the number of possible paths with greater length become increasingly difficult to count manually.

\section{Acknowledgments}

This material is based upon work supported by the National Research Foundation under grant numbers 89147 and 103530.

\section{References}

[1] C. Gordon, D. Webb, S. Wolpert, Bull. Am. Math. Soc. 27, 134 (1992).

[2] M. Kac, Am. Math. Monthly 73, 1 (1966).

[3] B. Gutkin, U. Smilansky, J. Phys. A Math. Gen. 34, 6061 (2001).

[4] P. Kurasov, M. Nowaczyk, J. Phys. A Math. Gen. 38, 4901 (2005).

[5] J. Griffith, Trans. Faraday Soc. 49, 345 (1953).

[6] S. Alexander, Phys. Rev. B 27, 1541 (1985).

[7] P. Exner, P. Šeba, in: Schrödinger Operators, Standard and Nonstandard, Dubna 1988, World Sci., 1989, p. 79.

[8] P. Kuchment, Waves Random Media 12, R1 (2002).

[9] J. von Below, J.A. Lubary, Results Math. 47, 199 (2005).

[10] P. Exner, J. Phys. A Math. Gen. 29, 87 (1996).

[11] K. Pankrashkin, Lett. Math. Phys. 77, 139 (2006).

[12] O. Post, in: Analysis on Graphs and Its Applications, Proc. Symposia in Pure Mathematics, Vol. 77, Am. Math. Soc., Providence (RI) 2008 p. 469.

[13] L. Baldassari, Europ. J. Appl. Math., 1 (2019). 